\title{
A Robust Digital Watermarking Technique for Image Contents based on DWT-DFRNT Multiple Transform Method
}

\author{
M. Kim ${ }^{1}$, D. $\mathrm{Li}^{2, *}$ and S. Hong ${ }^{1}$ \\ ${ }^{1}$ Department of Computer and Information Sciences, Towson University, \\ Towson, Maryland, USA \\ ${ }^{2}$ Department of Computer Science, University of Yanbian, \\ Yanji, China \\ leader1223@ybu.edu.cn \\ Abstract
}

Copyright protection of digital content has become one of impontant issues in digital content marketplace. Digital watermarking may be used as an effective method for identifying the copyright ownership of digital content against unauthorized use and distribution. In this paper, we propose a robust digital image watermarking algorithm based on the multiple transform method, discrete wavelet transform (DWT) and discrete fractional random transform (DFRNT). We adopt a two-dimensional (2D)barcode for hiding information and apply the block code encoding and generate a watermark through them. The generated watermark image is embedded into DWT-DFRNT using quantization technique in order to ensure robustness and imperceptibility of the Watermark. Experimental results present that our proposed algorithm has intproved the extraction performance by accurately extracting the hidden information in the $2 D$ barcodefrom the detected watermark. Also, combining the dual transform method, DWT and DKRNT, has improved the imperceptibility and robustness of the watermark agamst basic inage signal processing attacks.

Keywords: Digital Wafermarking, Discrete Wavelet Transform (DWT), Discrete Fractional Random Transform (DFRNT), 2D-barcode, Quantization

\section{Introduction}

With the phenomenal growth in digital content marketplace, illegal acts by unauthorized users to copy,edit, and distribute digital contents, which are copyrighted, are also increasing constantly. By the circulation of reproduced or manipulated illegal digital contents, the legal market is being influenced and copyright owners may lose the right for their contents. Thus, copyright protection and authentication of digital contents are emerging as serious problems in digital content marketplace [1,2]. Digital watermarking [3,4] can be used as an effective method for identifying the copyright ownership of digital content from illegal manipulation and distribution.

The basic idea of digital watermarking is embedding watermark data, which contains information about the copyright of the digital content, imperceptibly into the digital media content such as images, audio, and video $[3,5]$. The hidden watermark data can be used to authenticate the integrity of the original content [6] and it can be extracted in such a way that the embedding process is inversely applied to the watermarked content.

*Corresponding author (email: leader1223@ybu.edu.cn) 
Digital watermarking techniques can be classified into the spatial domain and frequency domain according to the domain used for embedding watermark. In the spatial domain based watermarking, watermark is embedded into digital contents in such a way that the process modifies the values of selected pixels, but it has weak robustness against common image signal processing and attacks such as noise, filtering, and compression, and may be easily destroyed by distortion [7]. In the frequency domain based watermarking, the process embeds a watermark into the selected portion of frequency domain by modifying the coefficients [8]. The frequency domain based watermarking is known as more robust and imperceptible technique than the spatial domain based watermarking, so the frequency domain is mostly used in recent watermarking methods $[5,9]$.

In the previous researches, many digital watermarking methods have been proposed. Cox et al., [10] proposed a secure watermarking algorithm using spread spectrum,Darpistaedter et al., [11] proposed a spatial watermarking algorithm by dividing an inage into blocks. Comparing to the early watermarking methods, many frequency doman based digital watermarking techniques, such as discrete cosine transform,(DCT) [12-14], DWT [15, 16], singular value decomposition (SVD) [17], and DFRAT 118, have been developed to improve their robustness and imperceptibility. In recent years, watermarking techniques based on dual transform domain such as DWT-DCT [19, 20], DWT-SVD [21], and SVD-DCT [22] have also been proposed.

In this paper, we propose a robust digitalimage watermarking algorithm based on the multiple transform method, DWT and DFRNT domainsusing 2D barcode in order to improve the extraction performance, imperceptibility and robustness of the watermark against image signal processing attacks such as inaged compression and noise adding.

\section{Related methods}

\subsection{D barcodes}

In the watermark generation process, we use 2D barcodes which have various information capacities and the self-error correction function. 2D barcodes widely used in various areas such as newspapers, pagazines, posters, TV, the internet, tickets, receipts, and advertisements. 2D bareodes retain information in two directions, horizontally and vertically, and thus the amount of recordable information is drastically greater than in a one-dimensional (1D) barcode. A 2D barcode is also applicable to digital content: A visible mark can be embedded intoddigital content such as a research article or an image so that it contains the information releyant to the content.

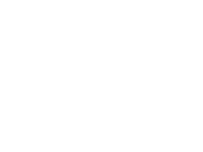

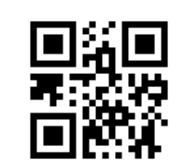

(a)

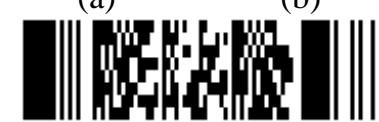

(c)

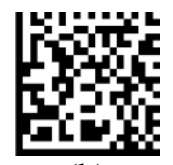

(b)

Figure 1. Types of 2D-barcode

Figure 1 shows some representative examples of 2D barcodes that have been released and frequently used: (a) the quick response (QR) code, (b) DataMatrix, and (c) PDF417. In 
different forms, all of them show a 2D barcode generated from the same information, the message "123456789." Among them, PDF417 is stack barcode, whereas the QR code and DataMatrix are based on the matrix method. The QR code holds the greatest amount of information, followed by DataMatrix and PDF417. Among the various types of 2D barcodes, the QR code is known to exhibit good performance in many respects, since the code size is small even if it contains a great deal of information, and the code can be scanned and read rapidly.

The information capacity and code size of 2D barcodes are dependent on the module size, error correction level, and types of encoding. Generally, the information capacity increases as the code size of the 2D barcode increases, but decreases as the error correction level rises. For example, a $21 \times 21$ cell QR code can contain 41 numbers or 25 alphanumeric data if the error correction level is low, but 17 numbers or 10 alphanumeric data if the error corredtion level is high. The information capacity of a $25 \times 25$ cell is about two times greater than that of a $21 \mathrm{x}$ 21 cell. Hence, a 2D barcode can be applied to the technology for digitat oontent copyright protection technology, such as forensic marking, thanks to the self-error correction function along with the maximized information capacity, minimized code region, and rapid code reading. This is because the information contained in a 2D barcode can be restored even after it is detected from compression, noise, and attack such as filtering, overcoming a certain range of error. In view of the different applications, a differentiated service can be provided, as the $2 \mathrm{D}$ barcode is detected when displaying content so that the information relevant to the content may be given on the screen.

\subsection{Multiple transform method}

The transform method used in the proposed system consists of a combination of DWT and DFRNT multiple transform in) order to ensure the imperceptibility and robustness of the watermark due to the Frequency decomposition ability of DWT that extracts robust coefficients and the unpredictable random characteristic of DFRNT.

For the multiple transform, first 2D-DWT [7, 23] is used and a host image signal is converted to a 2D signal to be used as the input for the 2D-DWT. The 2D-DWT-converted image signals can be decomposed into $\mathrm{H}(\mathrm{LH}), \mathrm{V}(\mathrm{HL})$, and $\mathrm{D}(\mathrm{HH})$, which have different frequency characteristics from one another. One time of 2D-DWT allows for the embedment of at least three watermarks. This not only robustly embeds the watermarks into a certain frequency band, but also allows the information about the copywriter and user, including the secondary copy writer or those with the neighboring copyright, to be additionally embedded into the coptent circulated by the copywriter of the content. This shows the pathways by which the contents are circulated and thereby enables effective multi-stage circulation tracking

DFRNT accepts the specific frequency coefficients generated by the 2D-DWT as the input data for the DFRNT, and randomly mixes the data by effecting various changes through the manipulation of the parameters. This leads to increased calculation complexity, so that the statistical characteristics of the data may not be understood by illegal users. The DFRNT [24] is generally performed in the method that follows.

Firstly, matrix $\mathrm{H}$ is generated using $\mathrm{P}$ generated as a random seed value, which is one of the parameters shown in Equation (1):

$$
\mathrm{H}=\frac{\mathrm{P}+\mathrm{P}^{\mathrm{T}}}{2}
$$


To generate an eigenvector from matrix H, SVD matrix decomposition is performed with respect to $\mathrm{H}$, as shown in Equation (2):

$\left[\mathrm{V}_{\mathrm{R}}, \mathrm{S}, \mathrm{U}\right]=\mathrm{SVD}(\mathrm{H})$

Here, the generated $V_{R}$ is the matrix composed of $\mathrm{N}$ orthogonal eigenvectors, as in Equation (3):

$\mathrm{V}_{\mathrm{R}}=\left[\mathrm{V}_{\mathrm{R} 1}, \mathrm{~V}_{\mathrm{R} 2}, \cdots, \mathrm{V}_{\mathrm{RN}}\right]$

Next, the $N \times N$ diagonal matrix $D_{\alpha}^{R}$ is generated using $\alpha$ and $m$, other parameters of DFRNT, as in Equation (4):

$D_{\alpha}^{R}=\operatorname{diag}\left[1, \exp \left(-i \frac{2 \pi \alpha}{m}\right), \cdots, \exp \left(-i \frac{2(N-1) \pi \alpha}{m}\right)\right]$

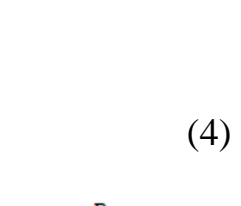

Then, $R^{\alpha}$ is calculated by Equation (5) using $V_{R}$ and $D_{\alpha}^{R}$. The calculated $R^{\alpha}$ and the DFRNT input signal $\mathrm{X}$ are substituted in Equation (6) to obtain $\mathrm{X}_{\mathrm{R}}$, the final output of the DRFNT:

$\mathrm{R}^{\alpha}=\mathrm{V}_{\mathrm{R}} \mathrm{D}_{\alpha}^{\mathrm{R}} \mathrm{V}_{\mathrm{R}}^{\mathrm{T}}$

$X_{R}=R^{\alpha} X\left(R^{\alpha}\right)^{T}$

In this way, DFRNT can fransform the input signals to arbitrary unpredictable signals with three parameters and restore them throughinverse transformation.

\section{Proposed Watermarking Teehnique}

\subsection{Watermark generation}

The watermark generation process is summarized by the steps as follows.

1) Generate a barcode containing information that is embedded into an image signal through a $2 \mathrm{D}$ barcode encoder

2) Put the generated barcode into the block code encoder that we designed for encoding it into a binary image.

3) Produce the watermark image.

Since the error correction of the $2 \mathrm{D}$ barcode is focused on the correction of bust error rather than random error, other possible errors other than bust error are corrected by such methods as block coding.

\subsection{Watermark embedding algorithm}

The watermark embedding algorithm is summarized as follows:

1) First, we generate the watermark image through the $2 \mathrm{D}$ barcode and block code encoding. 
2) The host image is decomposed into three sub-bands, H, V, and D, through the twolevel 2D-DWT. The 2D-DWT is performed by scanning the image signal in the $8 \times 8$ block unit, and some coefficients are chosen among the specified sub-band coefficients of each block according to the key table.

3) Next, the DFRNT is performed on the sub-band coefficients. And then, we embed the watermark image into the sub-band coefficient value using quantization technique.

4) Finally, we performed the inverse DFRNT (IDFRNT) and inverse DWT (IDWT).

5) As the final result, we obtain the watermarked image.

Figure 2 shows the steps in the watermark embedding algorithm.

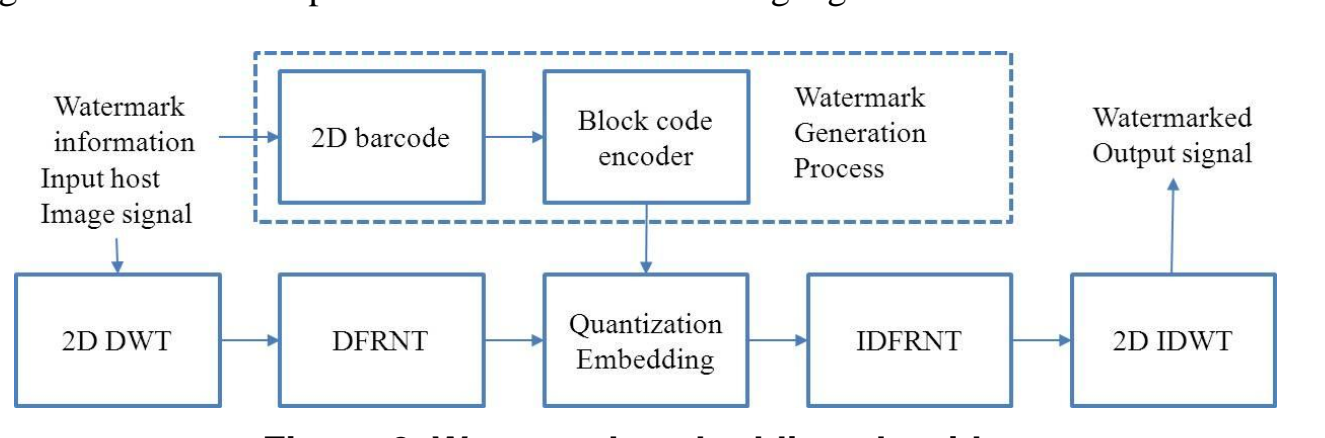

Figure 2. Watermark embedding algorithm

\subsection{Watermark extracting algorithm}

The watermark extraction process is performed in such a way that the embedding process is inversely applied to the watermarked image signal.

\section{Experimental Results}

In order to evaluate the performance of our proposed algorithm, we have performed the experiments with respect to the extraction performance, imperceptibility, and robustness of the watermark in various conditions including image compression and noise adding attacks.

We used $512 \times 512$ size standard gray level images as the sample host images and a $21 \times 21$ cell $\mathrm{QR}$ code, and $2 \times 2$ block code encoder.

For performing multiple transform, two-level 2D-DWT is performed to decompose the input image signal, $512 \times 512$ gray image, into the three sub-band frequencies of $\mathrm{H}, \mathrm{V}$, and $\mathrm{D}$. The 2D-DW $\mathrm{T}$ is performed by scanning the image signal in the $\mathrm{n} \times \mathrm{n}$ block unit until all pixels of the image signal are transformed. The size of scanning block is set to 8 , and the size of the sub-band coefficient matrix generated through performing the two-level 2D-DWT on the image of $512 \times 512$ size would be $64 \times 64$. Then, DFRNT is performed on each of sub-band frequencies. The default setting values for the parameters of the DFRNT function are set to: $\alpha$ $=0.01 ; \mathrm{m}=3$; and random seed $\mathrm{rs}=1$. The value of the quantization coefficient $\mathrm{Q}$ is determined to be in the range of 20 to 25 according to the quality demand of images.

In order to evaluate the imperceptibility and robustness, we compute the peak signal to noise ratio (PSNR) and Bit Error Rate (BER). BER value is calculated as follows:

$$
\mathrm{BER}=\frac{\sum_{\mathrm{i}=1}^{\mathrm{P} \times \mathrm{P}} \omega_{\mathrm{i}} \oplus \omega_{\mathrm{i}}^{*}}{\mathrm{P} \times \mathrm{P}} \times 100 \%
$$

The experimental result of the proposed watermark embedding algorithm without being 
attacked is shown in Table 1. The result verifies that the watermark is imperceptible and there are no visible differences between the two images by comparing the original image signal with the image signal after embedding the watermark into the $\mathrm{H}$ band of $2 \mathrm{D}-\mathrm{DWT}$. The $\mathrm{Q}$ value for embedding was set to 25 and the acquired PSNR is $40.01 \mathrm{~dB}$ for Lena image.

\section{Table 1. Image signals before and after embedding watermark}

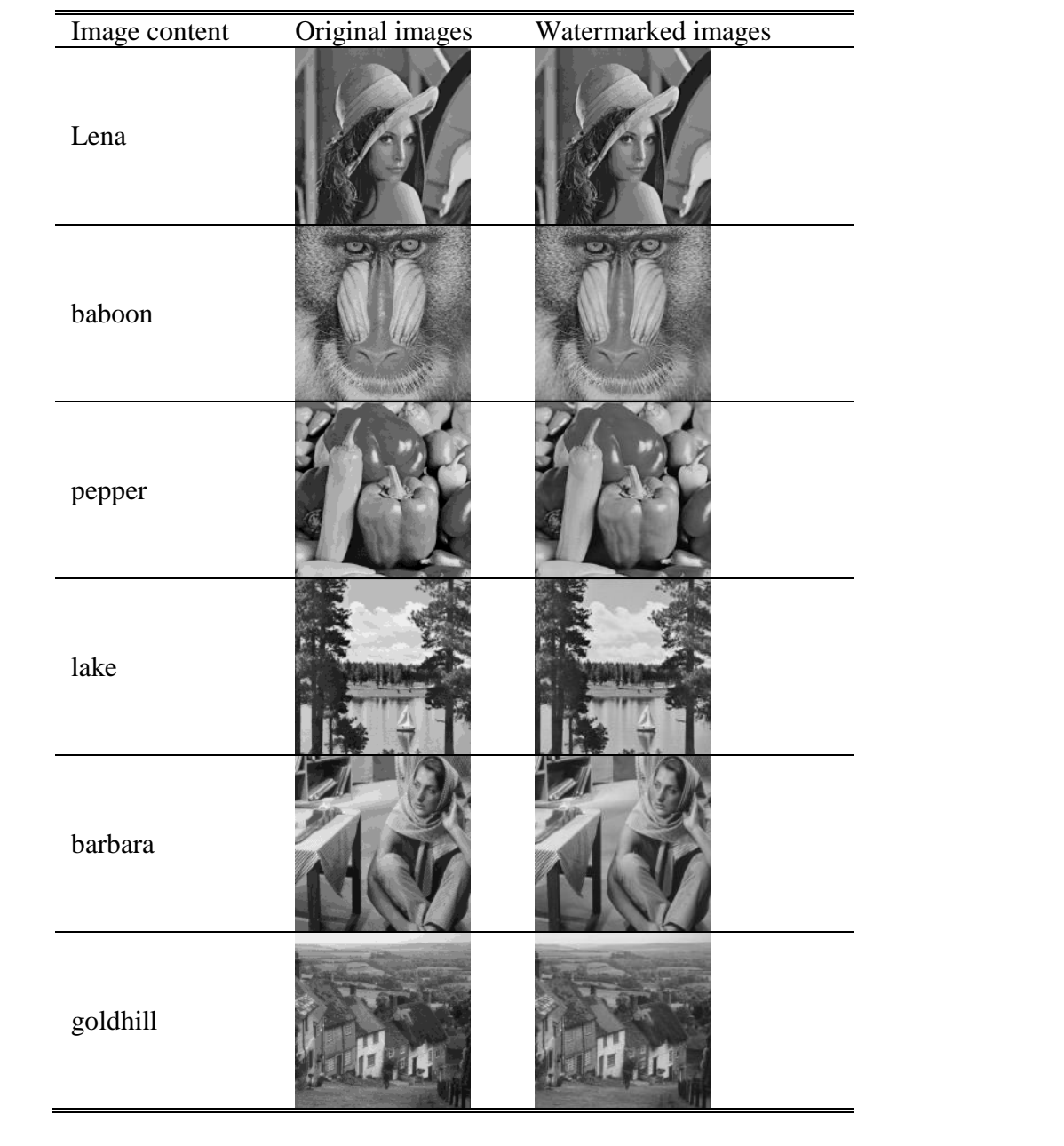

We eyaluated the extraction performance of the proposed watermarking algorithm by computing BER and the experimental results are shown in Figure 3. Figure 3 shows (a) the extracted watermark image from the watermarked Lena image, (b) the restored 2D barcode. In the experimental result, the restored $2 \mathrm{D}$ barcode gave that $\mathrm{BER}=0 \%$. This indicates that the 2D barcode was correctly restored.

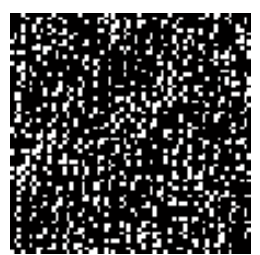

(a) Extracted watermark

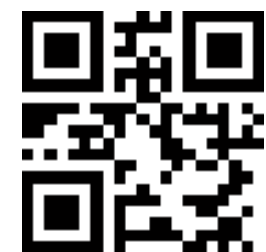

(b) Restored QR code

Figure 3. Extraction and restoration of 2D-barcode 
The PSNR values before and after embedding the watermark for the diverse standard images are given in Table 2. In the experimental result, the average PSNR of the test sample images was $40.22 \mathrm{~dB}$ and BER of the extracted watermark kept $0 \%$ in all the sample images.

Table 2. Conformance experiment of the watermarked images

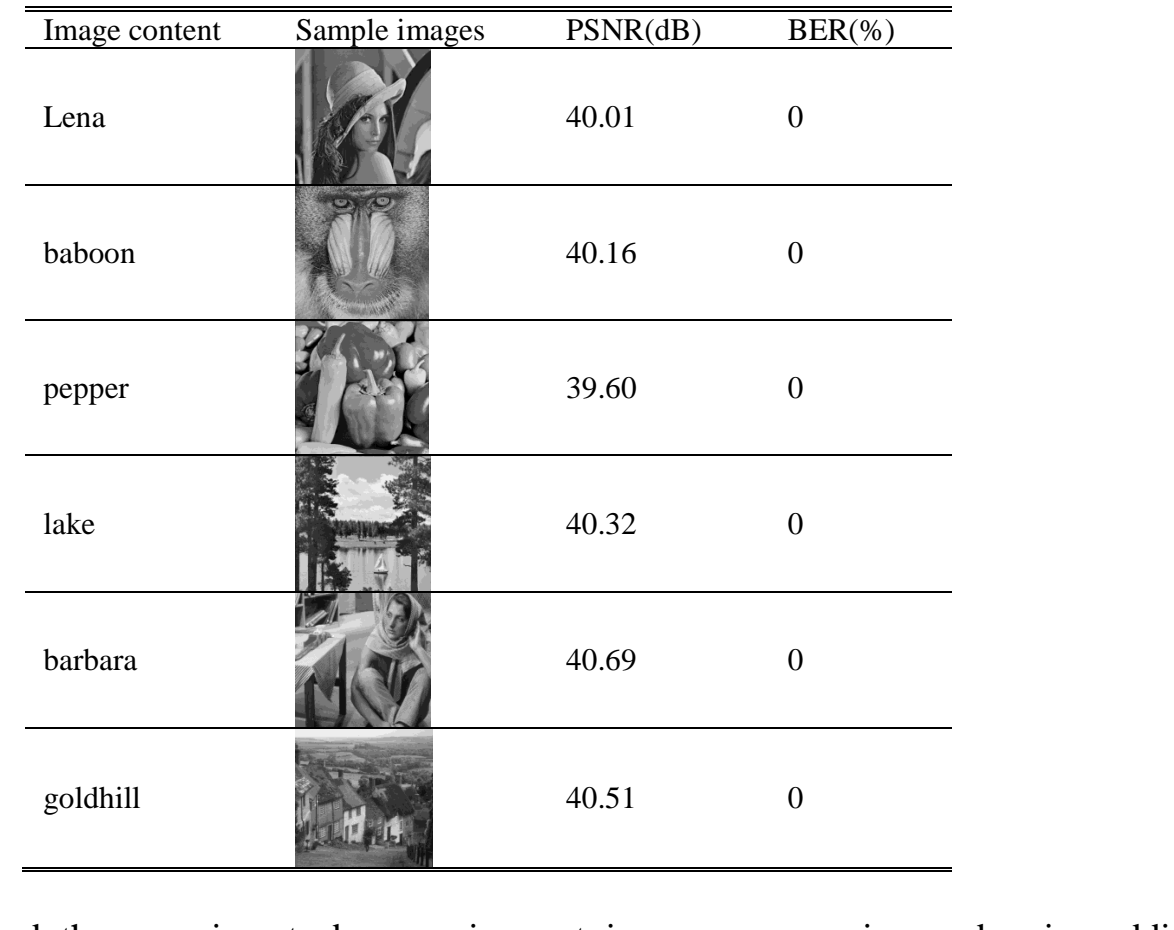

We performed the experiments by carrying out image compression and noise adding attacks, and the extracted and restored $Q R$ codes after being attacked are shown in Table 3 . In the case of compression attack, JPEG Quality Factor (QF) was set to 65. In the case of noise attack, Noise (g)denotes the Gaussian noise with mean set to 0 and variance set to 0.001 , and Noise(s) denotes the Salt \& Bepper noise with density set to 0.01 .

The experimental results verify that the proposed algorithm ensures the imperceptibility and robustness of the watermark.

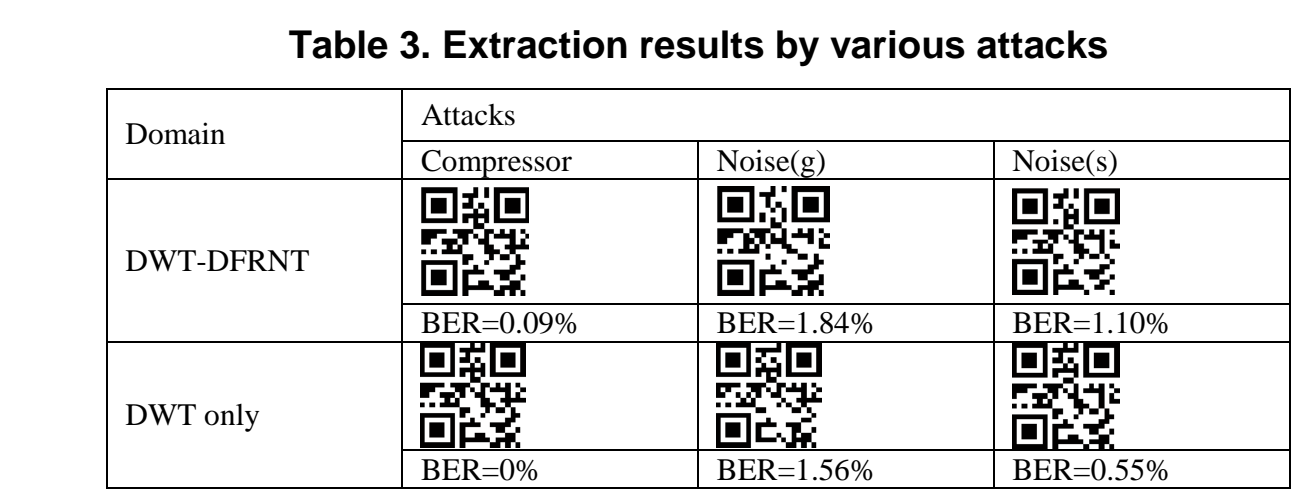

In addition, we evaluated the extraction performance of the proposed algorithm with reference to BER through comparing the DWT single domain with the DWT-DFRNT multiple transform domain. In the performed experiment, we computed the BER values the 
extracted watermark images fixing the PSNR at $40 \mathrm{~dB}$ after being attacked and compared them in the two types of domain.

As given in Table 3, the experimental results verify that while the DWT-DFRNT multiple transform domain has a similar level of extraction performance compare with that of the DWT single domain, and the 2D barcodes of both domains could be restored.

\section{Conclusions}

In this paper, we have proposed a robust digital watermarking technique for image contents based on DWT-DFRNT multiple transform method.

The watermark generation process has been implemented by using the $2 \mathrm{D}$ barcode and block encoding techniques in order to improve the extraction performance of the watermark. The watermark embedding process is completed by transforming the generated watermarks through the multiple transform which takes the benefits of the frequency decomposition ability of DWT and the inherent randomness of DFRNT. The experimental results presented that the proposed system has a good embedding/extraction performance. The embedded watermarks satisfied the imperceptibility and the quality of the watermarked images maintained at a high level, and the embedded 2D bareodes as watemarks could be perfectly restored. The proposed algorithm is also robustrgainst general image processing attacks such as image compression and noise adding.

\section{Acknowledgements}

This research project was supported by the National Natural Science Foundation of China (Grant No. 61262090).

\section{References}

[1] V. Bhat K, I. Sergupta and A. Das, “An adaptive audio watermarking based on the singular value decomposition in the wavelet domain") Digital Signal Processing, vol. 20, no 6, (2010), pp. 1547-1558.

[2] X.-Y. Wang and H. Zhao, "A Novel Synchronization Invariant Audio Watermarking Scheme Based on DWT and DCT”, IEEE Transactions on Signal Processing, vol. 54, no. 12, (2006), pp. 4835- 4840.

[3] I. J. Cox, M. L. Miller, J.M. G. Linnartz and T. Kalker, "A Review of of Watermarking Principles and Practices”, Digital SignalProcessing for Multimedia Systems by IEEE, (1999), pp. 461-485.

[4] I. J. Cox and M. Miller, "The first 50 years of electronic watermarking", Journal on Applied Signal Processing, Vol. 2002, No 2, (2002), pp. 126-132.

[5] H.-Y. Huang, C.-H. Yang and W.-H. Hsu, "A Video Watermarking Technique Based on Pseudo-3-D DCT and Quantization Index Modulation”, IEEE Transactions on Information Forensics and Security, vol. 5, no. 4, (2010), pp. 625-637.

[6] C.Busch, W. Funk and S. Wolthusen, "Digital watermarking: From concepts to real-time video applications", TEEE Transactions on Computer Graphics and Applications, vol. 19, no. 1, (1999), pp. 25-35.

[7] V. M. Potdar, S. Han and E. Chang, "A Survey of Digital Image Watermarking Techniques", Proceedings of the 3rd IEEE International Conference on Industrial Informatics, (2005), August; Perth, Australia.

[8] K. K. Sharma and D. K. Fageria, "Watermarking based on image decomposition using self-fractional Fourier functions", Journal of Optics, vol. 40, no. 2, (2011), pp. 45-50.

[9] A. Piva, M. Barni and F. Bartolini, "Copyright Protection of Digital Images by Means of Frequency Domain Watermarking”, Proceedings of SPIE Conference on Mathematics of Data/Image Coding, Compression, and Encryption, vol. 3456, (1998), July; San Diego, CA.

[10] I. J. Cox, Joe Kilian, F. T. Leighton and T. Shamoon, "Secure spread spectrum watermarking for multimedia," IEEE Transactions on Image Processing, vol. 6, (1997), pp. 1673-1687.

[11] V. Darmstaedter, J. f. Delaigle, J. J. Quisquater and B. Macq, "Low cost spatial watermarking", Computers \& Graphics, vol. 22, no. 4, (1998), pp. 417-424.

[12] W. C. Chu, "DCT-Based Image Watermarking Using Subsampling", IEEE Transactions on Multimedia, vol. 5, no. 1, (2003), pp. 34-38.

[13] F. Deng and B. Wang, "A novel technique for robust image watermarking in the DCT domain", Proceedings 
of the IEEE 2003 International Conference on Neural Networks and Signal Processing, vol. 2, (2003), December 14-17; Nanjing, China.

[14] V. Saxena, P. Khemka, A. Harsulkar and J. P. Gupta, "Performance analysis of color channel for DCT based image watermarking scheme", International Journal of Security and Its Applications, vol. 1, no .2, (2007), pp. 41-46

[15] S. Q. Wu, J. W. Huang and Y. Q. Shi, "Efficiently self-synchronized audio watermarking for assured audio data transmission", IEEE Transactions on Broadcasting, vol. 51, no. 1, (2005), pp. 69-76.

[16] D. Zhang, B. Wu, J. Sun and H. Huang, "A new robust watermarking algorithm based on DWT", 2nd International Congress on Image and Signal Processing, (2009), October 17-19; Tianjin, China.

[17] C. -C. Chang, P. Tsai and C. -C. Lin, "SVD-based digital image watermarking scheme", Pattern Recognition Letters, vol. 26, no. 10, (2005), pp. 1577-1586.

[18] Q. Guo and S. Liu, "Novel image fusion method based on discrete fractional random transform", Chinese Optics Letters, vol. 8, no. 7, (2010), pp. 656-660.

[19] A. Al-Haj, "Combined DWT-DCT Digital Image Watermarking”, Journal of Computer Science.vel. 3, no. 9, (2007), pp. 740-746.

[20] W. Liu and C. Zhao, "Digital watermarking for volume data based on 3D-DWT and 3D-DCT", Proceedings of the 2nd International Conference on Interaction Sciences: Inforniation Technology, Cuiture and Human, ACM, vol. 403, (2009), November 24-26; Seoul, Korea.

[21] Q. Li, C. Yuan and Y. Z. Zong, "Adaptive DWT-SVD domain image watermarking using human visual model", Proceedings of 9th International Conference on Ad anced Cómmunication Technology, vol. 3, (2007), February 12-14; Gangwon-do, Korea.

[22] B. Y. Lei, I. Y. Soon and Z. Li, "Blind and robust audio watermarking scheme based on SVD-DCT", Signal Processing, vol. 91, no. 8, (2011), pp. 1973-1984.

[23] M. J. Shensa, "The Discrete Wavelet Transform: Wedding the A Trous and Mallat Algorithms", IEEE Transactions on Signal Processing, vol. 40, no.10, (1992), pp. 2464-2482.

[24] Z. Liu, H. Zhao and S. Liu, “A discrete fractional random transform”, Optics Communications, vol. 255, no. 4, (2005), pp. 357-365.

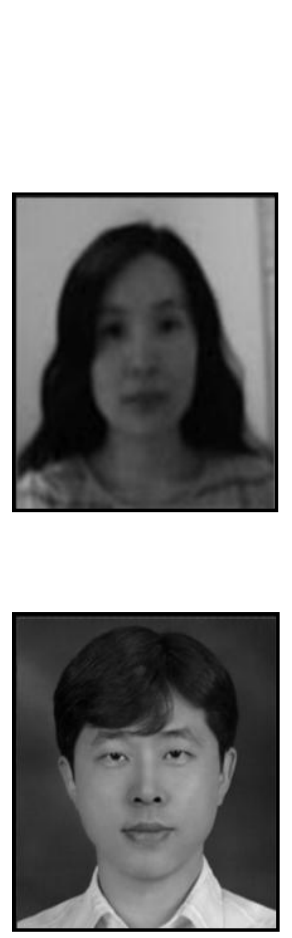

\section{D. $\mathbf{L i}$}

He received the Ph.D. degree from Sangmyung University, major in computer science. He is currently a professor in the department of Computer Science at Yanbian University in China. He is also a Principal Researcher at Copyright Protection Research Institute, Sangmyung University. His research interests are in the areas of copyright protection technology, digital watermarking, and digital forensic marking. 


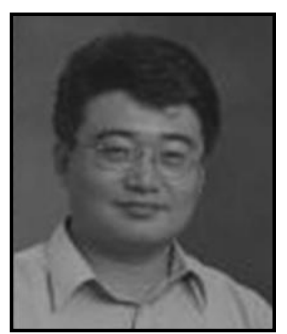

\section{S. Hong}

He received the MS degree in computer science and $\mathrm{PhD}$ degree in MIS from University of Texas at Dallas, major in E-Commerce. He is currently a professor in the department of Computer and Information Sciences at Towson University which is located in Maryland, USA. His research interests are in the areas of multi agent systems in B2B trading, electronic commerce, decision support systems, generic algorithm, digital watermarking.

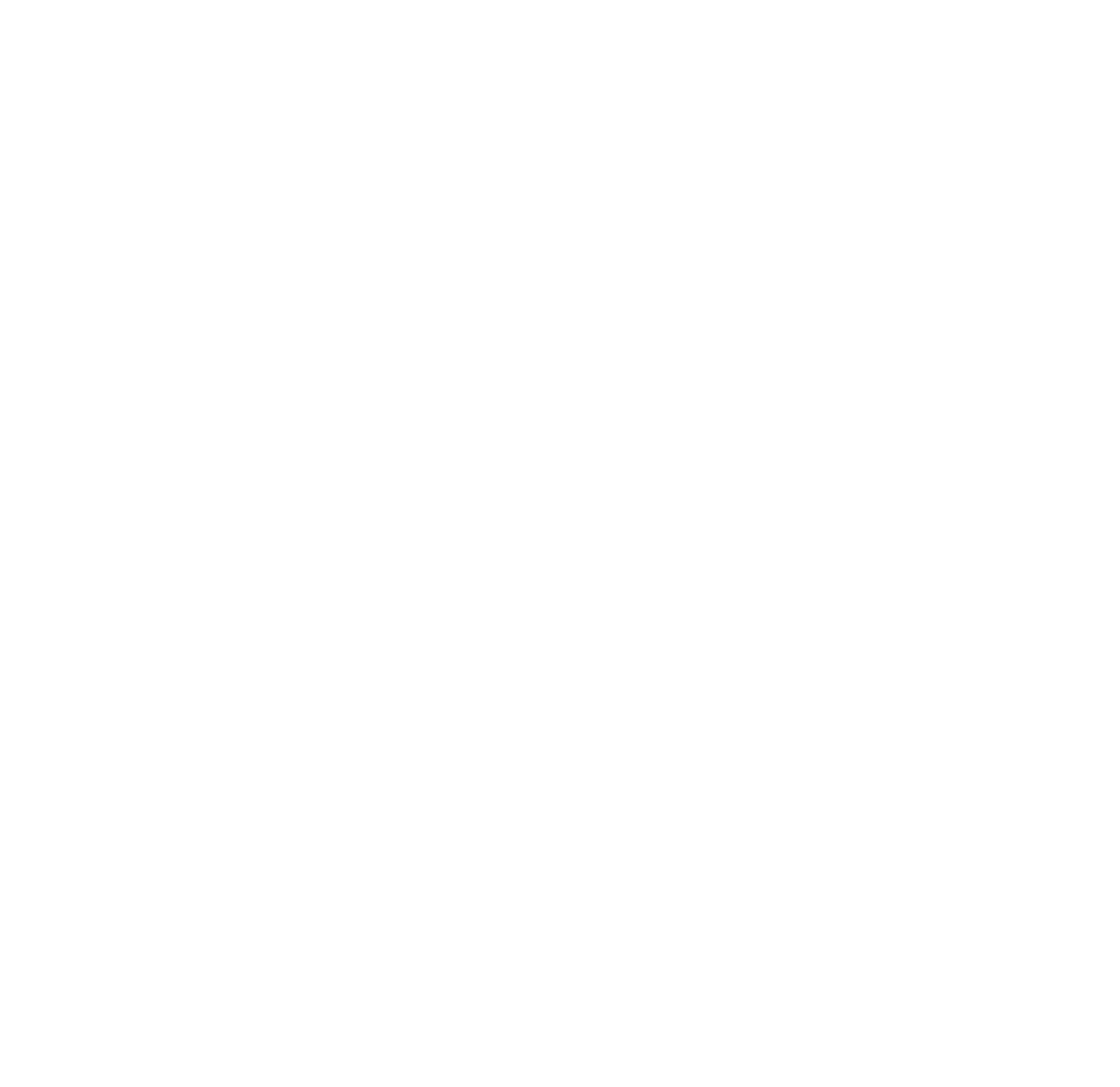

